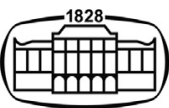

AKADÉMIAI KIADÓ

Journal of Psychedelic Studies

4 (2020) 3, 163-170

DOI:

$10.1556 / 2054.2020 .00136$

(C) 2020 The Author(s)

\section{RESEARCH ARTICLE}

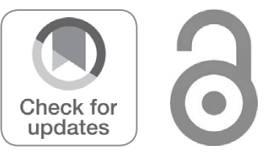

\title{
Tripping over the other: Could psychedelics increase empathy?
}

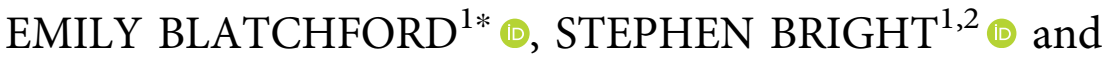 LIAM ENGEL ${ }^{1}$ @}

\author{
${ }^{1}$ School of Medical and Health Sciences, Edith Cowan University, Perth, Australia
}

${ }^{2}$ National Drug Research Institute, Curtin University, Perth, Australia

Received: February 28, 2020 • Accepted: July 05, 2020

Published online: September 5, 2020

\section{ABSTRACT}

There is increasing evidence that psychedelic-assisted psychotherapy is effective for a range of psychological conditions. There are likely numerous mechanisms of action that contribute to these clinical effects. One such mechanism of action might involve psychedelics increasing levels of empathic functioning. This paper synthesises research concerning the relationship between psychedelics and empathy, emphasising neuroscientific and clinical contexts. We conclude that neuropsychological and clinical evidence imply psychedelics could lead to increased empathic functioning. The effects of psychedelics on the 5-HT system, default mode network, neural connectivity and ego dissolution are implicated in these changes. Changes in empathic functioning also likely relate to increases in the personality trait of openness associated with psychedelic drug use, which is well documented. Increased empathic function likely has clinical implications, leading to increased social connectedness as well as prosocial attitudes and behaviours more broadly.

\section{KEYWORDS}

psychedelic, empathy, altruism, openness, default mode network, ego dissolution

\section{INTRODUCTION}

Classic psychedelics provide unique effects on human subjective experience (Bright \& Williams, 2018) by affecting the serotonin (or 5-hydroxytryptamine [5-HT]) system, primarily as $5-\mathrm{HT}_{2 \mathrm{~A}}$ receptor agonists (Forstmann \& Sagioglou, 2017). Examples of psychedelics include:

(i) psilocybin, commonly found in Psilocybe and Panaeolus species of mushrooms;

(ii) lysergic acid diethylamide (LSD), a synthetic ergotamine derivative;

(iii) dimethyltryptamine (including N,N-dimethyltryptamine [N,N-DMT] and 5-methoxy$\mathrm{N}, \mathrm{N}$-dimethyltryptamine [5-MeO-DMT] which are different alkaloids), found in a variety of different species in the plant and animal kingdoms;

(iv) ayahuasca, traditionally a combination of two plants, Psychotria viridis which contains N,N-DMT and Banisteriopsis caapi, containing monoamine oxidase inhibitors that make the N,N-DMT orally active; and

(v) mescaline, found primarily in Lophophora and Trichocereus species of cacti (Barbosa et al., 2016).

Psychedelics alter visual (e.g., altered colours and shapes) and psychological (e.g., mood and cognition) perception (Forstmann \& Sagioglou, 2017). The psychedelic-induced "state" is characterised as consisting of a sense that all things are one, feelings of ineffability, an intuitive belief that the experience has provided objective truth about reality or transcendence of time and space (Elsey, 2017). Other elements of the psychedelic-induced state may include creativity, enhanced self-reflection or ego dissolution (Elsey, 2017). Ego dissolution is defined as the feeling that one's self is disintegrating or that the border between one's self and the external world is dissolving (Lebedev et al., 2015). 
The potential benefits of psychedelics and psychedelicassisted psychotherapies are receiving increased attention due to a recent international resurgence in psychedelic research. Clinical and neuropsychological evidence suggests psychedelics may have capacity to facilitate social functioning. For example, Schmid and Liechti (2018) found that for participants who received $200 \mu \mathrm{g}$ of LSD, self-reported altruism ratings, as measured by the Persisting Effects Questionnaire, had significantly increased at 1- and 12month follow-ups. Furthermore, Griffiths et al. (2018) found that participants given a high dose of psilocybin exhibited significantly greater increases in prosocial attitudes and behaviours than participants given a low dose of psilocybin, measured by the Persisting Effects Questionnaire six months following the dose. There is also mounting evidence suggesting psychedelics might have therapeutic efficacy (for review, see Nichols, 2016). In particular, when psilocybin has been administered in a clinical context, it has produced clinical improvements in major depressive disorder (Carhart-Harris et al., 2018), anxiety (Griffiths et al., 2018; Ross et al., 2016), obsessive-compulsive disorder (Moreno, Wiegand, Taitano, \& Delgado, 2006), and addiction (Bogenschutz et al., 2015; Johnson, Garcia-Romeu, Cosimano, \& Griffiths, 2014; Johnson, Garcia-Romeu, \& Griffiths, 2017). Increases in empathy may contribute to the therapeutic action of psilocybin and other psychedelics.

Empathy forms a crucial component of human relationships and is an important predictor of a well-functioning society (Pokorny, Preller, Kometer, Dziobek, \& Vollenweider, 2017). It is hypothesised that there are two key components to empathy: (i) cognitive empathy, which is defined as being able to understand the emotional or mental state of another person without necessarily sharing the state; and (ii), emotional empathy, which is defined as being able to share the emotional state of another person (Pokorny et al., 2017). People with poor empathic functioning have difficulty understanding and sharing in reciprocal social interactions, which can lead them to being more vulnerable to social withdrawal, which increases the risk of developing a psychological disorder. Indeed, deficits in empathy have been reported among people with a variety of psychological conditions, including borderline personality disorder (Dziobek et al., 2011), bipolar disorder (Shamay-Tsoory, Harari, Szepsenwol, \& Levkovitz, 2009), psychopathy (Blair, 2005), and major depressive disorder (Cusi, MacQueen, Spreng, \& McKinnon, 2010). For example, Cusi et al. (2010) found that, in comparison to matched controls, patients with major depressive disorder reported lower levels of empathy.

Empathy also plays a fundamental role in all helping relationships, and is important for professionals, such as social workers and psychologists, who care for others in their work. Empathy allows practitioners to communicate empathic understanding whilst maintaining emotional resilience (Grant, 2014). Empathy is an essential component of the therapeutic alliance (Nienhuis et al., 2018), which is one of the strongest predictors of success within psychological therapy (Degeorge, 2008). In a meta-analysis of 59 studies which incorporated 3,599 clients, Elliot, Bohart,
Watson, and Greenberg (2011) found client, therapist and observer-rated empathy were moderately strong predictors of therapy outcome, mean weighted $r=0.31, P<0.001,95 \%$ CI $[0.28,0.34]$.

Psychedelic-assisted psychotherapy has been found to be effective for a range of psychological conditions including major depressive disorder (Carhart-Harris et al., 2018), anxiety (Griffiths et al., 2018; Ross et al., 2016), obsessivecompulsive disorder (Moreno et al., 2006), and addiction (Bogenschutz et al., 2015; Johnson et al., 2014; Johnson et al., 2017). Further, neuropsychological and clinical evidence implies psychedelics could lead to increased empathic functioning. The effects of psychedelics on the 5-HT system (Harmer et al., 2003), default mode network (Carhart-Harris et al., 2015; Muthukumaraswamy et al., 2013), neural connectivity (Cabanis et al., 2013) and ego dissolution (Tagliazucchi et al., 2016) are implicated in these changes. Therefore, this paper aimed to consider if the positive outcomes that have been observed in recent studies of psychedelic-assisted psychotherapy (for review, see Bright \& Williams, 2018) are due to increased empathy produced by the psychedelic drug.

\section{CLINICAL EVIDENCE}

Several researchers have recently found links between psychedelic use and empathy (Dolder, Schmid, Müller, Borgwardt, \& Liechti et al., 2016; Grob et al., 2011; Lerner \& Lyvers, 2006; Mason, Mischler, Uthaug, \& Kuypers et al., 2019; Pokorny et al., 2017). Lerner and Lyvers (2006) conducted a survey of 183 participants from Australia and Israel with diverse histories of drug use. They found people who had used psychedelics scored higher in emotional empathy than people who had not, as measured by the Emotional Empathic Tendency Scale. While these studies were not clinical trials, a feeling of oneness with all things could explain the higher emotional empathy scores. That is, the feeling of no boundaries between the self and the world, and that all things were connected could, like the experience of a close relationship, lead to broader empathic concern. Mason et al. (2019) considered the impact of psilocybin consumed in a retreat setting. The authors tested participants' empathy using the Multifaceted Empathy Test (MET) three times the night prior to psilocybin consumption $(N=55)$, the morning after consumption $(N=50)$ and seven days following $(N=22)$. The MET required participants to view photographs of people and rate how much they were feeling for each individual. Mason et al. (2019) found that emotional empathy increased the morning following use and that emotional empathy was retained seven days later, noting that these differences in empathy were correlated with wellbeing. Dolder et al. (2016) randomly administered single oral doses of $100 \mu \mathrm{g}$ of LSD to 24 participants, and $200 \mu \mathrm{g}$ of LSD to 16 other participants. They found LSD enhanced emotional empathy, as measured by the MET. Dolder et al. (2016) found empathogenic effects were significantly greater in participants receiving the $200 \mu \mathrm{g}$ 
dose. Similarly, Pokorny et al. (2017) conducted a randomised controlled trial (RCT) where $0.215 \mathrm{mg} / \mathrm{kg}$ of psilocybin was administered to 32 healthy human participants. Pokorny et al. found increases in emotional empathy, as measured by the MET $160 \mathrm{~min}$ after psilocybin administration. Grob et al. (2011) administered $0.2 \mathrm{mg} / \mathrm{kg}$ of psilocybin to advanced-stage cancer patients in a double-blind, placebo-controlled design. They found empathic rapport increased through strengthened relationships with friends and family following the psilocybin dose. Increases in emotional empathy following LSD and psilocybin administration and increased empathy among people who use psychedelics provide direct evidence that the administration of psychedelics in a clinical context leads to increased empathy.

Levels of empathy have been found to correlate with the personality dimension of openness (Jolliffe \& Farrington, 2006). Openness is one of the big five personality factors and refers to broad-minded tolerance of others' values and viewpoints, aesthetic appreciation, sensitivity, imagination and fantasy (MacLean, Johnson, \& Griffiths, 2011). It has been found to increase following psychedelic administration in experimental and longitudinal studies. For example, MacLean et al. (2011) found significant increases in openness following a high psilocybin dose of $30 \mathrm{mg} / 70 \mathrm{~kg}$. This increase in openness was sustained at the 16-month followup. This was the first study to demonstrate personality changes in healthy adults following a single, experimentally manipulated event. The relationship between psychedelics and openness has been further explored by Barbosa et al. (2016), who found that União do Vegetal members in Brazil who had experienced ayahuasca had higher levels of openness than matched controls with no ayahuasca experience. Additionally, Lebedev et al. (2016) observed significant increases in openness two weeks after administration of $75 \mu \mathrm{g}$ of LSD to participants. As researchers have found higher openness among people who use psychedelics, and increased openness following psychedelic administration, openness might be an important factor to consider concerning the relationship between psychedelics and empathy.

\section{NEUROSCIENCE, EMPATHY, PSYCHEDELICS AND MECHANISMS OF ACTION}

There are three inter-related neural mechanisms by which psychedelics might exert an effect on empathy: (i) an interaction with the $5-\mathrm{HT}$ system as $5-\mathrm{HT}_{2 \mathrm{~A}}$ receptor agonists; (ii) the deactivation of the default mode network $(\mathrm{DMN})$; and (iii), increasing neural connectivity.

\section{Psychedelics and the serotonin system}

Psychedelic drugs could plausibly enhance empathic functioning as 5-HT agonists because 5-HT both plays a role in moral judgement and behaviour and modulates empathic responses to the emotional states of others (Harmer et al., 2003). For instance, 5-HT manipulations have been found to influence the detection of emotion in faces. Harmer et al.
(2003) conducted an RCT and found that healthy participants who were intravenously administered citalopram, a selective serotonin reuptake inhibitor (SSRI), detected facial expressions of happiness and fear faster and more accurately than participants who received a placebo. Additionally, injection of 5-HT agonists such as 5-HT creatinine sulphate has promoted the release of oxytocin and vasopressin (Jørgensen, Riis, Knigge, Kjaer, \& Warberg, 2003), both of which are neuropeptides strongly implicated in empathy (Crockett, Clark, Hauser, \& Robbins, 2010). For instance, a single dose of intranasally administered oxytocin significantly increased correct responses to the Reading the Mind in the Eyes Test (Domes, Heinrichs, Michel, Berger, \& Herpertz, 2007), which measures empathic ability to infer the emotional states of others (Baron-Cohen, Wheelwright, Hill, Raste, \& Plumb, 2001). The effect of vasopressin may be explained by its ability to modulate the activation of the right amygdala, which is involved in empathic functioning. For example, in an RCT where intranasal vasopressin or a placebo was administered, participants were asked to explain how they felt in response to pictorial stimuli depicting people in different social and emotional situations. Activity in the right amygdala increased during the processing of pictures for participants who received vasopressin but not the placebo (Brunnlieb, Münte, Tempelmann, \& Heldmann, 2013). Psychedelics may offer similar outcomes to SSRIs, although through different neurochemical processes. Psychedelics are agonists with a high affinity for the $5-\mathrm{HT}_{2 \mathrm{~A}}$ receptor, and SSRIs block the 5-HT reuptake mechanism so that over time there is more 5-HT at the synapse. Interestingly, many patients in trials of psilocybin for treatmentresistant depression reported that conventionally prescribed treatments, such as SSRIs, reinforced a sense of disconnection with others (Watts, Day, Krzanowski, Nutt, \& CarhartHarris, 2017). The 5-HT system plays a role in moderating empathic responses, and psychedelics agonise $5-\mathrm{HT}_{2 \mathrm{~A}}$ receptors. This relationship between psychedelics and the 5HT system suggests that psychedelics could play a role in the moderation of empathic functioning.

\section{Deactivation of the default mode network}

The DMN consists of a set of brain regions that are active during "rest", or during less demanding tasks (Metin et al., 2015). Some of these regions include the medial prefrontal cortex, the posterior cingulate cortex (PCC), and the temporo-parietal junction (Andrews-Hanna, Smallwood, \& Spreng, 2014). As task-related attentional demands increase, activity within the DMN is decreased. This is referred to as attenuation (Metin et al., 2015).

The DMN is involved in self-referential processing and rumination (Nejad, Fossati, \& Lemogne, 2013). Self-referential processing refers to the cognitive process of relating information to the self, and rumination is a form of selfreferential processing characterised by repetitive thinking and a focus on negative mood states (Nejad et al., 2013). Major depression is associated with a bias towards negative emotional processing and increased self-focus and 
rumination (Nejad et al., 2013). Moberly and Watkins (2008) found that a bidirectional relationship exists between ruminative self-focus and negative effect. One example of rumination is reflecting on one's self in the past (Speth et al., 2016). Such rumination could involve going over something that has happened in the past and cannot be changed, which is unhelpful as it can lead to negative emotions and unproductive behaviours. Speth et al. (2016) found intravenous administration of $75 \mu \mathrm{g}$ of LSD led to a reduction in the number of linguistic references to mental time travel to the past. Mental time travel refers to individuals mentally projecting themselves backwards and forwards in time, to recollect components of previous autobiographical episodes or to imagine future experiences (Speth et al., 2016). Consequently, Speth et al.'s results suggest that psychedelics might decrease activity in the DMN, leading to decreased rumination and focus on the self. In turn, such attenuation of the DMN may encourage empathy by increasing thought about others. By discouraging self-centric thinking, psychedelic-induced attenuation of the DMN may also encourage empathy so that people may be more able to appreciate benefits for others.

The DMN also plays a role in helping individuals develop a coherent sense of self or ego (Carhart-Harris et al., 2014). Therefore, decreased activity in the DMN has also been linked to ego dissolution (Carhart-Harris et al., 2015; Muthukumaraswamy et al., 2013), which is the feeling that the border between one's self and the external world is dissolving (Lebedev et al., 2015). Ego dissolution is one explanation for how psychedelic use could increase empathy. If the boundary between the self and others becomes blurred through ego dissolution, people might better understand or share the emotional state of another person, leading to an increase in empathic behaviour.

Researchers have examined brain signals through measuring neural magnetic fields and blood flow in the brain and have shown that psychedelics decrease alpha power in the PCC and in the DMN more broadly. These changes in brain function have correlated with reports of ego dissolution and may consequently contribute to empathy because individuals can feel more connected with others. For example, following intravenous administration of $2 \mathrm{mg}$ of psilocybin to participants, Muthukumaraswamy et al. (2013) conducted magnetoencephalography (MEG) and found that the magnitude of alpha power decreased in the PCC, which forms a central part of the DMN. These decreases correlated with ratings of the item, "I experienced a disintegration of my 'self or 'ego"' (Muthukumaraswamy et al., 2013). Further, in an RCT by Carhart-Harris et al. (2015) in which participants were injected with $75 \mu \mathrm{g}$ of LSD, functional magnetic resonance imaging (fMRI) and MEG scans showed that LSD decreased activity in the DMN. Specifically, there were differences in cerebral blood flow, resting state functional connectivity, and blood oxygen level-dependent signal variance within the DMN after LSD administration compared to the placebo. Carhart-Harris et al. found that this DMN disintegration correlated with ego dissolution. Correlations between ego dissolution and changes in brain function imply that psychedelics blur the boundary between the self and other, and that individuals who use psychedelics might share more due to valuation of benefits to others in addition to benefits to the self.

\section{Increased neural connectivity}

Decreased activity in the DMN appears to be associated with increased global connectivity among high-level association cortices rich in $5-\mathrm{HT}_{2 \mathrm{~A}}$ receptors and the thalamus. This has been demonstrated by fMRI following LSD administration (Tagliazucchi et al., 2016). High-level association cortices are areas of the cortex that are located between visual, auditory and somatosensory cortices (Arslan, 2016). Because these cortices are associated with self-awareness (Craig, 2011) and emotional processing (Luan Phan, Wager, Taylor, \& Liberzon, 2002), it is plausible to assume that increased neural connectivity resulting from psychedelic use might facilitate empathy. Self-awareness refers to the ability to recognise oneself as an individual separate from the environment and other individuals (Silvia \& Duval, 2001). These increases in neural connectivity might facilitate similar psychological effects.

Similar to the decreased activity in the DMN, increases in global connectivity among cortical association regions have also been selectively correlated with subjective ego dissolution ratings (Tagliazucchi et al., 2016). Two areas between which LSD has shown to increase functional connectivity are the bilateral temporo-parietal junction and the bilateral insular cortex (Tagliazucchi et al., 2016). The bilateral insular cortex is related to self-awareness (Craig, 2011) and the processing of emotional information (Luan Phan et al., 2002). This increased neural connectivity in the bilateral temporo-parietal junction and the bilateral insular cortex, observed by fMRI, has been correlated with ego dissolution (Tagliazucchi et al., 2016). Luan Phan et al. (2002) conducted a meta-analysis of functional neuroimaging studies and found that emotional tasks with cognitive demand particularly involved the insula. More recently, Cabanis et al. (2013) administered an attributional bias task during fMRI scanning where participants were asked to decide whether situations described in sentences were caused by themselves or by the other person involved (e.g., "The waitress ignores you at the bar"). Bilateral insular activation was found to correlate with awareness of personal agency in negative situations (Cabanis et al., 2013). That is, increased bilateral insular activity was observed when participants assumed and accepted responsibility for causing a negative social event, thus demonstrating a non-self-serving bias.

Neuroimaging studies have found that the parts of the brain activated by thinking about the self are different from the parts of the brain activated by thoughts concerning the future self (Ersner-Hershfield, Wimmer, \& Knutson, 2009). Interestingly, the same part of the brain associated with the future self is associated with thoughts about others, namely the cortical midline structures of the medial prefrontal cortex (mPFC) and rostral anterior cingulate (rACC). The relationship between psychedelics and the $\mathrm{mPFC}$ and $\mathrm{rACC}$ 
will likely be an important area for future research concerning the relationship between psychedelics and empathy.

As psychedelics activate and connect brain regions associated with self-awareness and the processing of emotional information, which in turn leads to less selfcentric thinking, it is plausible that people who use psychedelics may exhibit enhanced empathic functioning. There is correlational, experimental and longitudinal evidence that provides direct links between psychedelic use and empathic functioning.

\section{CLINICAL IMPLICATIONS}

The possibility that psychedelics enhance empathy has implications for applications of psychedelic-assisted psychotherapy. The increased empathy occasioned by a psychedelic experience could be a direct mechanism of action in producing some of the positive outcomes reported from psychedelic-assisted psychotherapy. In this respect, psychedelicassisted psychotherapy may be useful for psychological conditions associated with deficits in empathy (Blair, 2005; Cusi et al., 2010; Dziobek et al., 2011; Shamay-Tsoory et al., 2009). As the therapeutic alliance is key to good clinical outcomes (Degeorge, 2008) and empathy is a key part of the therapeutic alliance (Nienhuis et al., 2018), increased empathy occasioned by a psychedelic experience could also provide an indirect mechanism of action in psychedelicassisted psychotherapy by enhancing therapeutic alliance. For example, some training protocols for psychedelic-assisted psychotherapy have required therapists to consume psychedelics in order to learn how to administer these drugs (Multidisciplinary Association for Psychedelic Studies, 2020). These experiences may increase the levels of therapists' empathy and thus improve therapeutic outcomes via strengthening of the therapeutic alliance.

The link between psychedelics and empathy also has implications for prosocial behaviour. Reduced social connectivity can precipitate and perpetuate a number of psychological conditions, particularly depression (Beck \& Alford, 2009; Hari, 2019). Watts et al. (2017) investigated whether psilocybin-assisted psychotherapy for treatmentresistant depression was effective in a qualitative study and explored the mechanisms of action by interviewing participants 6 months following treatment (Watts et al., 2017). Responses were analysed for consistent themes and all patients who endorsed the treatment's effectiveness referenced renewed connections with others. Similarly, Carhart-Harris and Goodwin (2017) found that people who used psychedelics showed significant increases in social connectedness two weeks after a psychedelic dose. Enhanced connections with others following psychedelic use might produce greater concern for others and contribute to psychedelics' influence on altruism and prosocial behaviour (Schmid \& Liechti, 2018; Griffiths et al., 2018). Empathy facilitates emotional awareness and reduces self-centricity, which in turn, could lead to prosocial behaviour and social connectivity, decreasing symptoms of depression as a result. Such increased empathy could lead to prosocial behaviour by altering core beliefs about the self, others, the world, purpose and meaning more broadly.

\section{CONCLUSION}

Both neuropsychological and clinical evidence can be used to suggest that psychedelics could lead to changes in empathic functioning and increase prosocial behaviour. Increases in empathic function and behaviour could be one mechanism of action that contributes to the positive effects of psychedelic-assisted psychotherapy. Empathy is implicated in a wide range of mental health conditions and psychedelics may have a similarly wide range of applications. Future research should consider how different types of psychedelics compare in their influence on empathic functioning as well as the comparable influence of different use contexts (i.e. recreational, spiritual, clinical). Greater longitudinal data is also needed to consider the impact of psychedelic use on empathy over time. We also encourage legislators to consider the potential negative impacts of psychedelic prohibition which undermines both the undertaking of psychedelic research and access to pharmacotherapies that could improve both individual quality of life and social cohesion.

Conflict of interest: No specific funding was used to support this project and there are no conflicts of interest to disclose.

\section{ACKNOWLEDGEMENTS}

We would like to thank Ross Hollett for his supervision of the project from which this review has been drawn.

\section{REFERENCES}

Andrews-Hanna, J. R., Smallwood, J., \& Spreng, R. N. (2014). The default network and self-generated thought: Component processes, dynamic control, and clinical relevance. Annals of the New York Academy of Sciences, 1316(1), 29-52. https://doi.org/ $10.1111 /$ nyas. 12360.

Arslan, O. E. (2016). Computational basis of neural elements. In M. Puri, Y. Pathak, V. K. Sutariya, S. Tipparaju, \& W. Moreno (Eds.), Artificial neural network for substance design, delivery and disposition. Retrieved from https://www.elsevier.com/ books.

Barbosa, P. C. R., Strassman, R. J., de Silveira, D. X., Areco, K., Hoy, R., Pommy, J., et al. (2016). Psychological and neuropsychological assessment of regular hoasca users, Comprehensive Psychiatry, 71, 95-105.

Baron-Cohen, S., Wheelwright, S., Hill, J., Raste, Y., \& Plumb, I. (2001). The "Reading the Mind in the Eyes" test, revised 
version: A study with normal adults, and adults with asperger syndrome or high-functioning autism. Journal of Child Psychology and Psychiatry, 42(2), 241-251. https://doi.org/10.1023/ A:1015497629971.

Beck, A. T., \& Alford, B. A. (2009). Depression: Causes and treatment. University of Pennsylvania Press.

Blair, R. J. R. (2005). Responding to the emotions of others: Dissociating forms of empathy through the study of typical and psychiatric populations. Consciousness and Cognition, 14(4), 698-718. https://doi.org/10.1016/j.concog.2005.06.004.

Bogenschutz, M. P., Forechimes, A. A., Pommy, J. A., Wilcox, C. E., Barbosa, P. C. R., \& Strassman, R. J. (2015). Psilocybin-assisted treatment for alcohol dependence: A proof-of-concept study. Journal of Psychopharmacology, 29(3), 289-299. https://doi.org/ 10.1177/0269881114565144.

Bright, S., \& Williams, M. (2018). Should Australian psychology consider enhancing psychotherapeutic interventions with psychedelic drugs? A call for research. Australian Psychologist, 53(6), 467-476.

Brunnlieb, C., Münte, T. F., Tempelmann, C., \& Heldmann, M. (2013). Vasopressin modulates neural responses related to emotional stimuli in the right amygdala. Brain Research, 1499, 29-42. https://doi.org/10.1016/j.brainres.2013.01.009.

Cabanis, M., Pyka, M., Mehl, S., Müller, B. W., Loos-Jankowiak, S., Winterer, G., et al. (2013). The precuneus and the insula in selfattributional processes. Cognitive, Affective \& Behavioural Neuroscience, 13(2), 330-345. https://doi.org/10.3758/s13415012-0143-5.

Carhart-Harris, R. L., Bolstridge, M., Day, C. M. J., Rucker, J., Watts, R., Erritzoe, D. E., et al. (2018). Psilocybin with psychological support for treatment-resistant depression: Sixmonth follow-up. Journal of Psychopharmacology, 235(2), 399408. https://doi.org/10.1007/s00213-017-4771-x.

Carhart-Harris, R. L., \& Goodwin, G. M. (2017). The therapeutic potential of psychedelic substances: Past, present and future. Journal of Neuropsychopharmacology. 42(11), 2105-2113. https://doi.org/10.1038/npp.2017.84.

Carhart-Harris, R. L., Leech, R., Hellyer, P. J., Shanahan, M., Feilding, A., Tagliazucchi, E., et al. (2014). The entropic brain: A theory of conscious states informed by neuroimaging research with psychedelic substances. Frontiers in Human Neuroscience, 8(20), 1-22. https://doi.org/10.3389/fnhum.2014. 00020 .

Carhart-Harris, R. L., Muthukumaraswamy, S., Roseman, L., Kaelen, M., Droog, W., Murphy, K., et al. (2015). Neural correlates of the LSD experience revealed by multimodal neuroimaging. Proceedings of the National Academy of Sciences of the United States of America, 113(17), 4853-4858. https://doi.org/10.1073/ pnas.1518377113.

Craig, A. D. (2011). Significance of the insula for the evolution of human awareness of feelings from the body. Annals of the New York Academy of Sciences, 1225(1), 72-82. https://doi.org/10. 1111/j.1749-6632.2011.05990.x.

Crockett, M. J., Clark, L., Hauser, M. D., \& Robbins, T. W. (2010). Serotonin selectively influences moral judgment and behavior through effects on harm aversion. Proceedings of the National
Academy of Sciences of the United States of America,107(40), 17433-17438. https://doi.org/10.1073/pnas.1009396107.

Cusi, A. M., MacQueen, G. M., Spreng, R. N., \& McKinnon, M. C. (2011). Altered empathic responding in major depressive disorder: Relation to symptom severity, illness burden, and psychosocial outcome. Psychiatry Research, 188(2), 231-236. https://doi.org/10.1016/j.psychres.2011.04.013.

Degeorge, J. (2008). Empathy and the therapeutic alliance: Their relationship to each other and to outcome in cognitive-behavioral therapy for generalized anxiety disorder. (Master's thesis, University of Massachussetts, Amherst, United States). Retrieved from https://scholarworks.umass.edu/theses/179/.

Dolder, P. C., Schmid, Y., Müller, F., Borgwardt, S., \& Liechti, M. E. (2016). LSD acutely impairs fear recognition and enhances emotional empathy and sociality. Journal of Neuropsychopharmacology, 41(11), 2638-2646. https://doi.org/10. 1038/npp.2016.82.

Domes, G., Heinrichs, M., Michel, A., Berger, C., \& Herpertz, S. C. (2007). Oxytocin improves "Mind-Reading" in humans. Society of Biological Psychiatry, 61(6), 731-733. https://doi.org/10.1016/ j.biopsych.2006.07.015.

Dziobek, I., Preissler, S., Grozdanovic, Z., Heuser, I., Heekeren, H. R., \& Roepke, S. (2011). Neuronal correlates of altered empathy and social cognition in borderline personality disorder. NeuroImage, 57(2), 539-548. https://doi.org/10.1016/j.neuroimage. 2011.05.005.

Elliot, R., Bohart, A. C., Watson, J. C., \& Greenberg, L. S. (2011). Empathy. Psychotherapy, 48(1), 43-49. https://doi.org/10.1037/ a0022187.

Elsey, J. W. B. (2017). Psychedelic substance use in healthy individuals: A review of benefits, costs, and implications for substance policy. Substance Science Policy and Law, 3, 1-11. https://doi.org/10.1177/2050324517723232.

Ersner-Hershfield, H., Winmer, G., \& Knutson B. (2009). Saving for the future self: Neural measures of future self-continuity predict temporal discounting. Social Cognitive and Affective Neuroscience, 4(1): 85-92. https://doi.org/10.1093/scan/ nsn042.

Forstmann, M., \& Sagioglou, C. (2017). Lifetime experience with (classic) psychedelics predicts pro-environmental behavior through an increase in nature relatedness. Journal of Psychopharmacology, 31(8), 1-14. https://doi.org/10. 11770269881117714049.

Grant, L. (2014). Hearts and minds: Aspects of empathy and wellbeing in social work students. Social Work Education, 33(3), 338-352. https://doi.org/10.1080/02615479.2013.805191.

Griffiths, R. R., Johnson, M. W., Richards, W. A., Richards, B. D., Jesse, R., MacLean, K. A., et al. (2018). Psilocybin-occasioned mystical-type experience in combination with meditation and other spiritual practices produces enduring positive changes in psychological functioning and in trait measures of prosocial attitudes and behaviors. Journal of Psychopharmacology, 32(1), 49-69.

Grob, C. S., Danforth, A. L., Chopra, G. S., Hagerty, M., McKay, C. R., Halberstadt, A. L., et al. (2011) Pilot study of psilocybin treatment for anxiety in patients with advanced-stage cancer. 
Archives of General Psychiatry, 68(1), 71-78. https://doi.org/10. 1001/archgenpsychiatry.2010.116.

Hari, J. (2019). Lost connections: Uncovering the real causes of depression-and the unexpected solutions. Bloomsbury Publishing Plc.

Harmer, C. J., Bhagwagar, Z., Perrett, D., Völlm, B. A., Cowen, P. J., \& G. M. Goodwin. (2003). Acute SSRI administration affects the processing of social cues in healthy volunteers. Journal of Neuropsychopharmacology, 28(1), 148-152. https://doi.org/10. 1038/sj.npp.1300004.

Johnson, M. W., Garcia-Romeu, A., Cosimano, M. P., \& Griffiths, R. R. (2014). Pilot study of the 5-HT2AR agonist psilocybin in the treatment of tobacco addiction. Journal of Psychopharmacology, 28(11), 983-992. https://doi.org/10.1177/0269881114548296.

Johnson, M. W., Garcia-Romeu, A., \& Griffiths, R. R. (2017). Longterm follow-up of psilocybin-facilitated smoking cessation. The American Journal of Substance and Alcohol Abuse, 43(1), 55-60. https://doi.org/10.3109/00952990.2016.1170135.

Jolliffe, D., \& Farrington, D. (2006). Development and validation of the basic empathy scale. Journal of Adolescence, 29(4), 589-611. https://doi.org/10.1016/j.adolescence.2005.08.010.

Jørgensen, H., Riis, M., Knigge, U., Kjaer, J., \& Warberg, J. (2003). Serotonin receptors involved in vasopressin and oxytocin secretion. Journal of Neuroendocrinology, 15(3), 242-249. https://doi.org/10.1046/j.1365-2826.2003.00978.x.

Lebedev, A. V., Lövdén, M., Rosenthal, G., Feilding, A., Nutt, D. J., \& Carhart-Harris, R. L. (2015). Finding the self by losing the self: Neural correlates of ego-dissolution under psilocybin. Human Brain Mapping, 36(8), 3137-3153. https://doi.org/10. 1002/hbm.22833.

Lerner, M., \& Lyvers, M. (2006). Values and beliefs of psychedelic substance users: A cross-cultural study. Journal of Psychoactive Substances, 38(2), 143-147. https://doi.org/10.1080/02791072. 2006.10399838.

Luan Phan, K., Wager, T., Taylor, S. F., \& Liberzon, I. (2002). Functional neuroanatomy of emotion: A meta-analysis of emotion activation studies in PET and fMRI. NeuroImage, 16(2), 331-348. https://doi.org/10.1006/nimg.2002.1087.

MacLean, K. A., Johnson, M. W., \& Griffiths, R. R. (2011). Mystical experiences occasioned by the hallucinogen psilocybin lead to increases in the personality domain of openness. Journal of Psychopharmacology, 25(11), 1453-1461. https://doi.org/10. $1177 / 0269881111420188$.

Mason, N., Mischler, E., Uthaug, M., \& Kuypers, K. (2019) Subacute effects of psilocybin on empathy, creative thinking and subjective wellbeing. Journal of Psychoactive Drugs, 51(2), 123134, https://doi.org/10.1080/02791072.2019.1580804.

Metin, B., Krebs, R. M., Wiersema, J. R., Verguts, T., Gasthuys, R., van der Meere, J. J., et al. (2015). Dysfunctional modulation of default mode network activity in attention deficit/hyperactivity disorder. Journal of Abnormal Psychology, 124(1), 208-214. https://doi.org/10.1037/abn0000013.

Moberly, N. J., \& Watkins, E. R. (2008). Ruminative self-focus and negative affect: An experience sampling study. Journal of Abnormal Psychology, 117(2), 314-323. https://doi.org/10.1037/ 0021-843X.117.2.314.
Moreno, F. A., Wiegand, C. B., Taitano, E. K., \& Delgado, P. L. (2006). Safety, tolerability, and efficacy of psilocybin in 9 patients with obsessive-compulsive disorder. Journal of Clinical Psychiatry, 67(11), 1735-1740. https://doi.org/10.1016/S00843970(08)70820-X.

Multidisciplinary Association for Psychedelic Studies (2020). A phase 1 placebo-controlled, double-blind, Multi-site crossover study to assess psychological effects of MDMA when administered to healthy volunteers. Retrieved 28 June 2020 fromhttps://mapscontent.s3-us-west-1.amazonaws.com/pdfs/ MT1+Santized +for +Website + Protocol + A5V2+Clean +08APR2020.pdf.

Muthukumaraswamy, S. D., Carhart-Harris, R. L., Moran, R. J., Brookes, M. J., Williams, T. M., Erritzoe, D., et al. (2013). Broadband cortical desynchronization underlies the human psychedelic state. Journal of Neuroscience, 33(38), 15171-15183. https://doi.org/10.1523/JNEUROSCI.2063-13.2013.

Nejad, A. B., Fossati, P., \& Lemogne, C. (2013). Self-referential processing, rumination, and cortical midline structures in major depression. Frontiers in Human Neuroscience, 7(666), 666. https://doi.org/10.3389/fnhum.2013.00666.

Nichols, D. E. (2016). Psychedelics. Pharmacological Reviews, 68(2), 264-355. https://doi.org/10.1177/0269881111420188.

Nienhuis, J. B., Owen, J., Valentine, J. C., Black, S. W., Halford, T. C., Parazak, S. E., et al. (2018). Therapeutic alliance, empathy, and genuineness in individual adult psychotherapy: A metaanalytic review. Psychotherapy Research, 28(4), 593-605. https://doi.org/10.1080/10503307.2016.1204023.

Pokorny, T., Preller, K. H., Kometer, M., Dziobek, I., \& Vollenweider, F. X. (2017). Effect of psilocybin on empathy and moral decision-making. The International Journal of Neuropsychopharmacology, 20(9), 747-757. https://doi.org/10.1093/ ijnp/pyx047.

Ross, S., Bossis, A., Guss, J., Agin-Liebes, G., Malone, T., Cohen, B., et al. (2016). Rapid and sustained symptom reduction following psilocybin treatment for anxiety and depression in patients with life-threatening cancer: A randomized controlled trial. Journal of Psychopharmacology, 30(12), 1165-1180.

Schmid, Y., \& Liechti, M. E. (2018). Long-lasting subjective effects of LSD in normal subjects. Journal of Psychopharmacology, 235(2), 535-545. https://doi.org/10.1007/s00213-017-4733-3.

Shamay-Tsoory, S., Harari, H., Szepsenwol, O., \& Levkovitz, Y. (2009). Neuropsychological evidence of impaired cognitive empathy in euthymic bipolar disorder. Journal of Neuropsychiatry and Clinical Neurosciences, 21(1), 59-67. https://doi.org/ 10.1176/appi.neuropsych.21.1.59.

Silvia, P. J., \& Duval, T. S. (2001). Objective self-awareness theory: Recent progress and enduring problems. Personality and Social Psychology Review, 5(3), 230-241. https://doi.org/10.1207/ S15327957PSPR0503_4.

Speth, J., Speth, C., Kaelen, M., Schloerscheidt, A. M., Feilding, A., Nutt, D. J., et al. (2016). Decreased mental time travel to the past correlates with default-mode network disintegration under lysergic acid diethylamide. Journal of Psychopharmacology, 30(4), 344-353. https://doi.org/10.1177/0269881116628430. 
Tagliazucchi, E., Roseman, L., Kaelen, M., Csaba, O., Muthukumaraswamy, S. D., Murphy, K., et al. (2016). Increased global functional connectivity correlates with LSD-induced ego dissolution. Current Biology, 26, 1043-1050. https://doi.org/10. 1016/j.cub.2016.02.010.
Watts, R. D. C., Day, C., Krzanowski, J., Nutt, D. J., \& CarhartHarris, R. L. (2017). Patients' accounts of increased 'connection' and 'acceptance' after psilocybin for treatment-resistant depression. Journal of Humanistic Psychology 55(5), 520-564. https://doi.org/10.3389/fnhum.2016.00269. 